Supporting Information for

\title{
Assembly of Chiral Cluster-Based Metal-Organic Frameworks and the Chirality Memory Effect during their Disassembly
}

\author{
Guocheng Deng, Boon K. Teo* and Nanfeng Zheng*
}

Collaborative Innovation Center of Chemistry for Energy Materials, State Key Laboratory for Physical Chemistry of Solid Surfaces, National \& Local Joint Engineering Research Center of Preparation Technology of Nanomaterials, College of Chemistry and Chemical Engineering, Xiamen University, Xiamen 361005, China

*Corresponding Authors. Email: nfzheng@xmu.edu.cn, boonkteo@xmu.edu.cn 


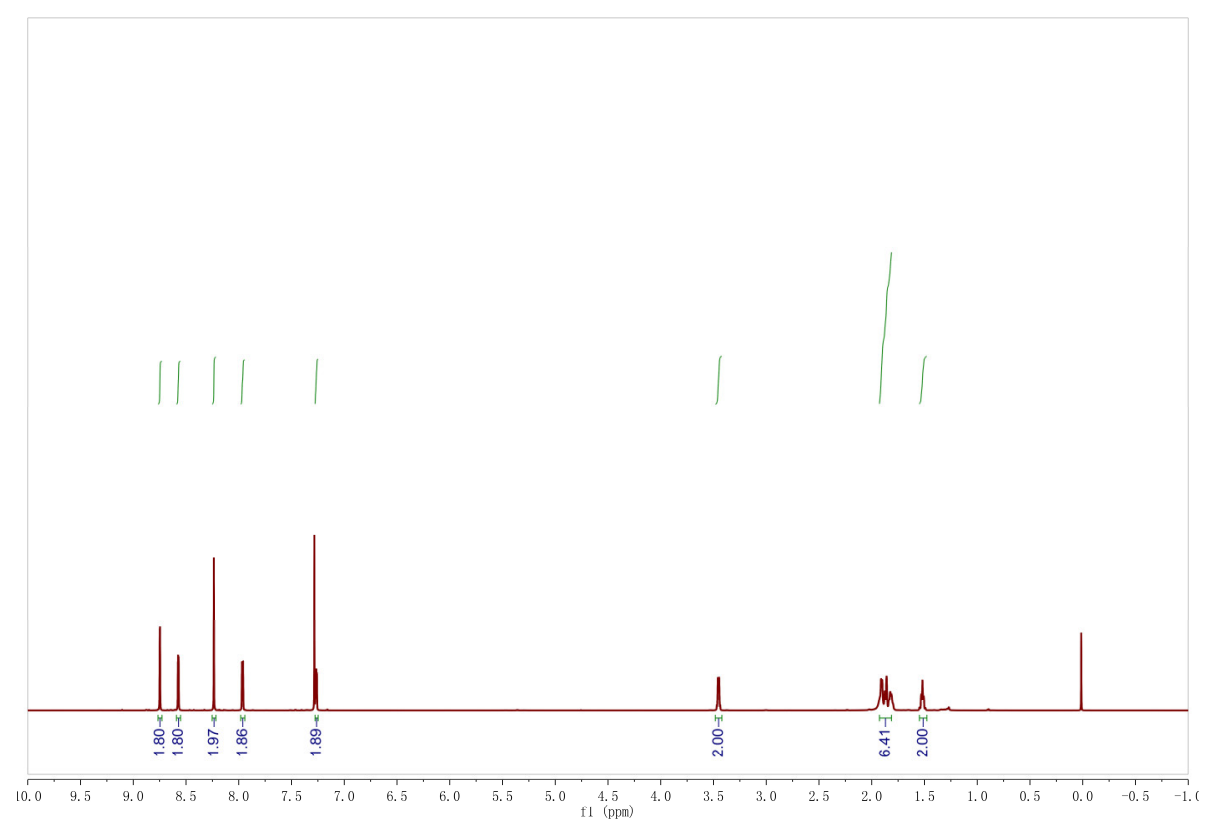

Figure S1. ${ }^{1} \mathrm{H}$ NMR spectrum of $\mathbf{L S}$ in $\mathrm{CDCl}_{3}$.

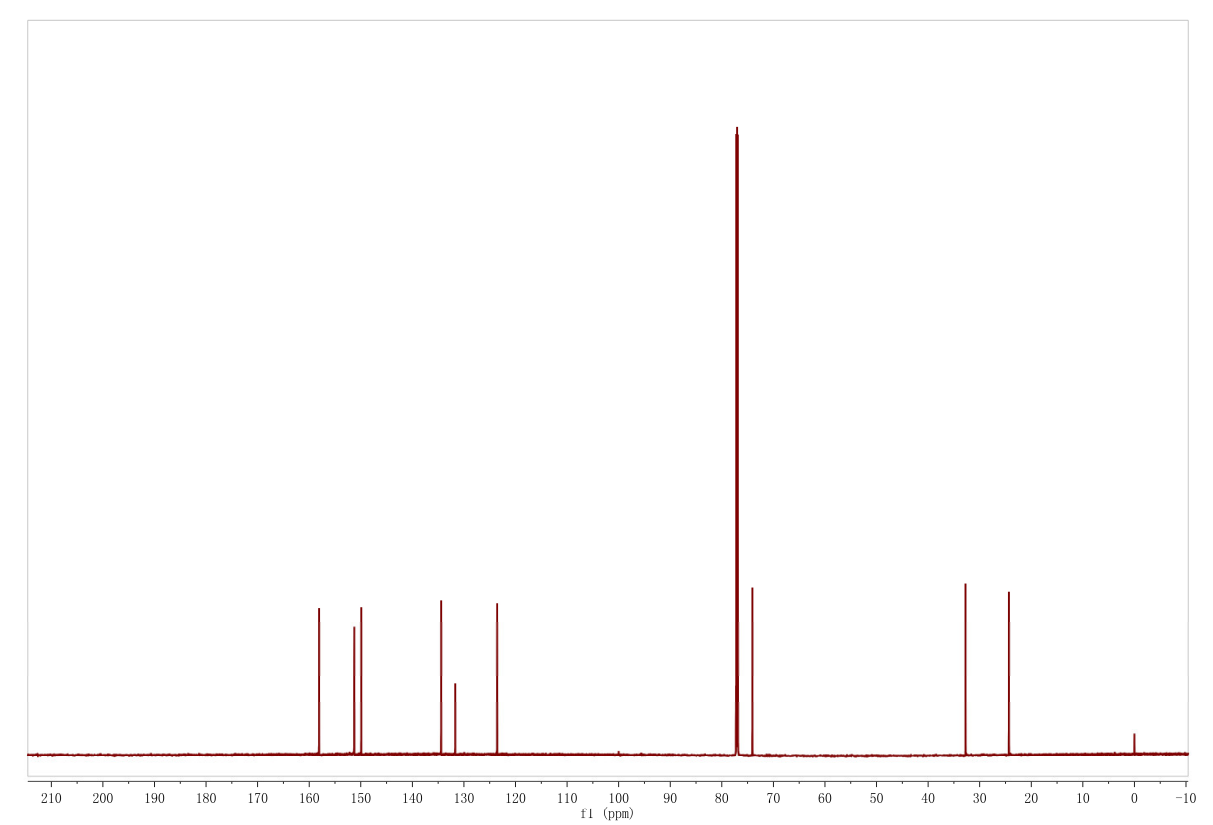

Figure S2. ${ }^{13} \mathrm{C}$ NMR spectrum of $\mathbf{L S}$ in $\mathrm{CDCl}_{3}$. 


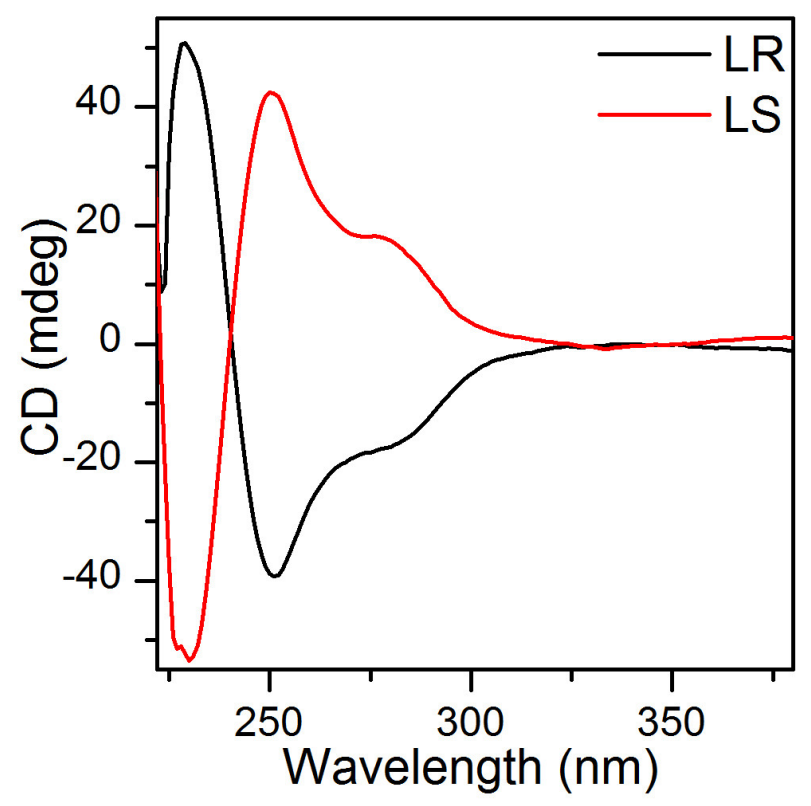

Figure S3. CD spectra of LR and LS in dichloromethane solution.

a

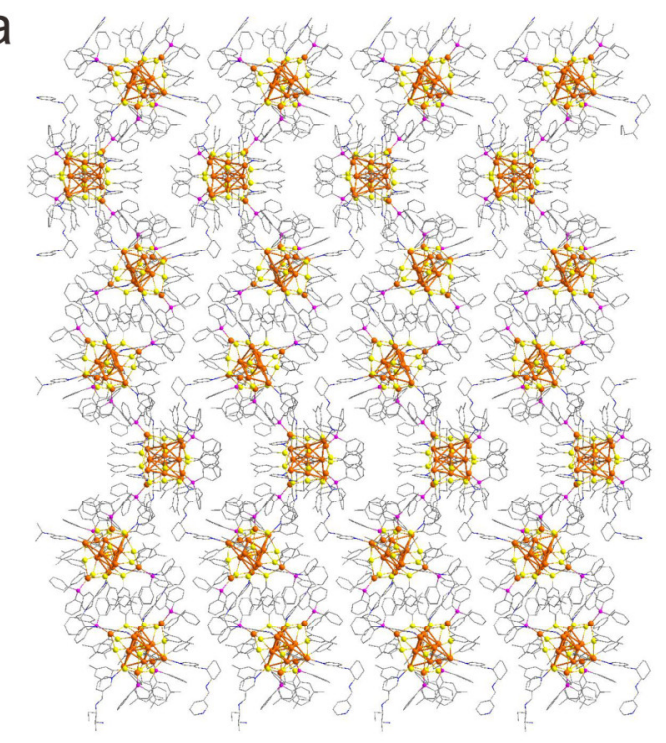

$\mathrm{b}$

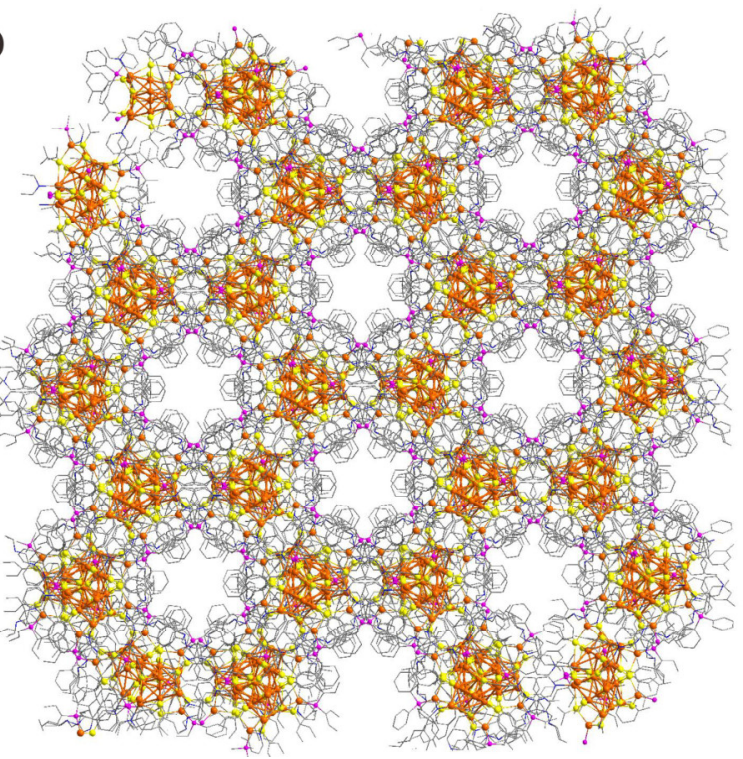

Figure S4. Crystal structure of Ag14-LS viewed along $b$ (a) and $c$ axis (b). Color legend: orange sphere, Ag; yellow sphere, S; pink sphere, P; blue sphere, N; grey sphere, C. All hydrogen and fluorine atoms are omitted for clarity. 


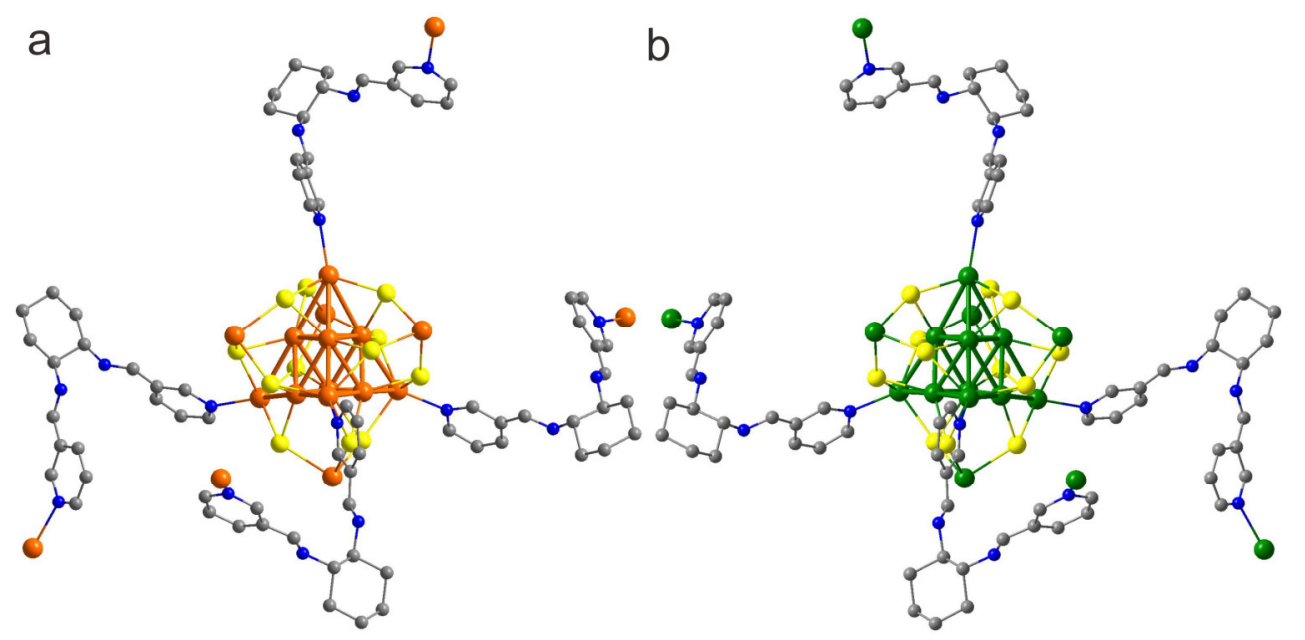

Figure S5. The structures of the building blocks of $\mathbf{A g}_{14}-\mathbf{L S}$ (a) and Ag14-LR (b). Color legend: orange and green sphere, Ag; yellow sphere, S; blue sphere, N; grey sphere, C.

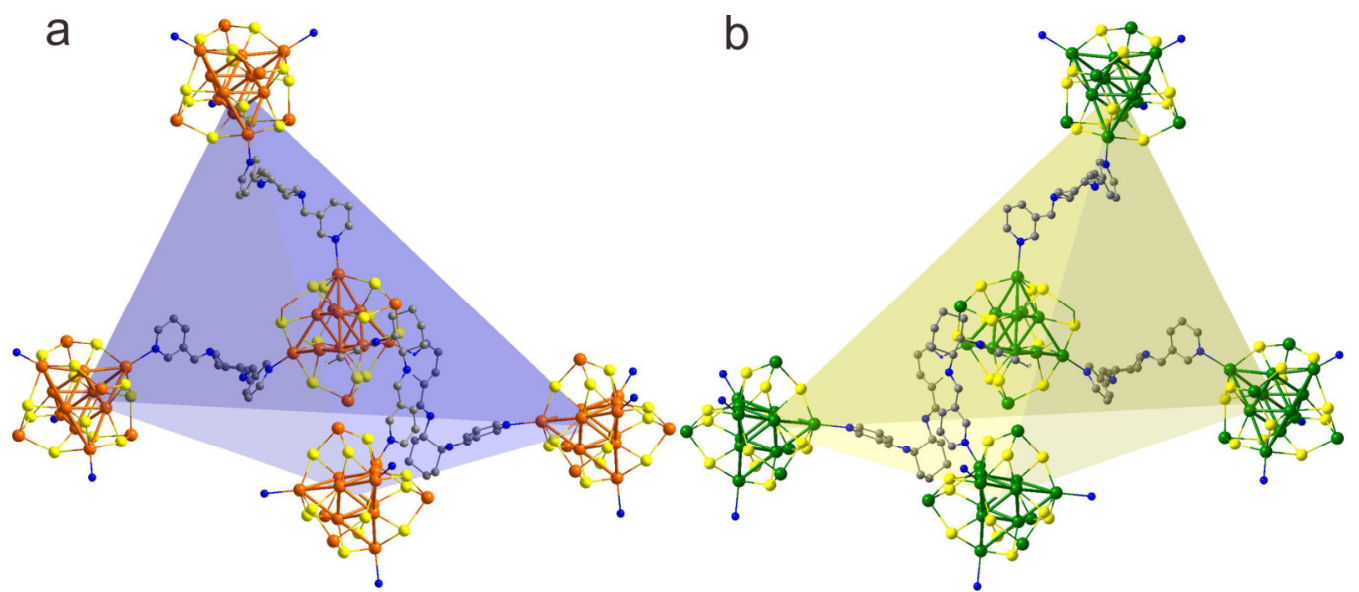

Figure S6. Distorted tetrahedron structures form by the Ag14 building blocks in Ag14-LS (a) and Ag14-LR (b). Color legend: orange and green sphere, Ag; yellow sphere, S; blue sphere, $\mathrm{N}$; grey sphere, $\mathrm{C}$. 


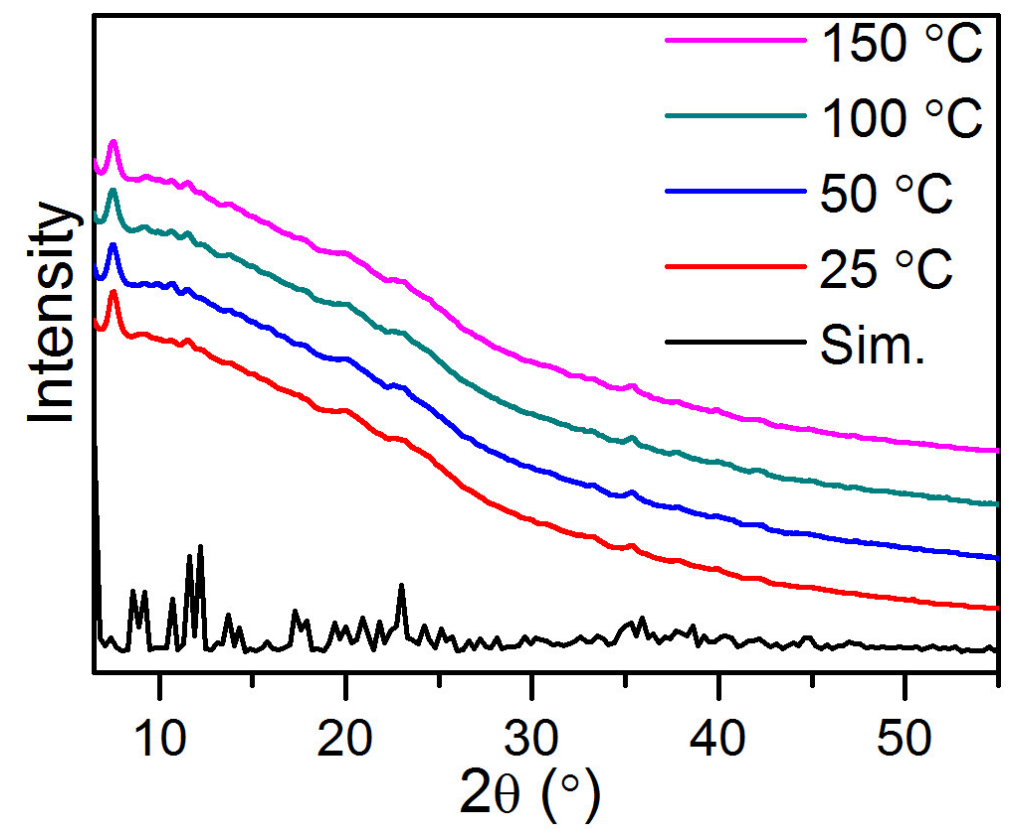

Figure S7. PXRD of $\mathbf{A g} 14-\mathbf{L R}$ from $25^{\circ} \mathrm{C}$ to $150{ }^{\circ} \mathrm{C}$.

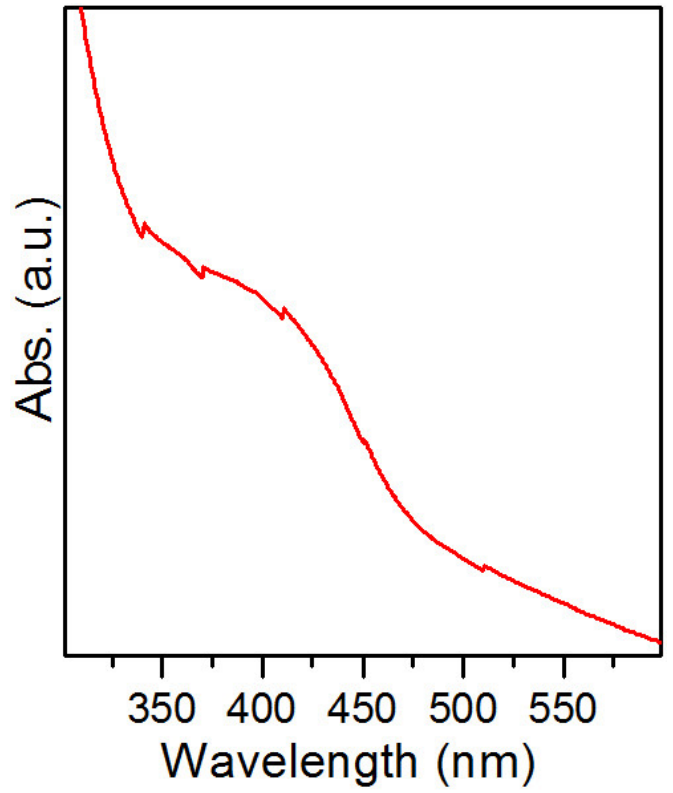

Figure S8. Solid state UV-vis spectrum of $\mathbf{A g}_{14-\mathbf{L S}}$ (crystals). 


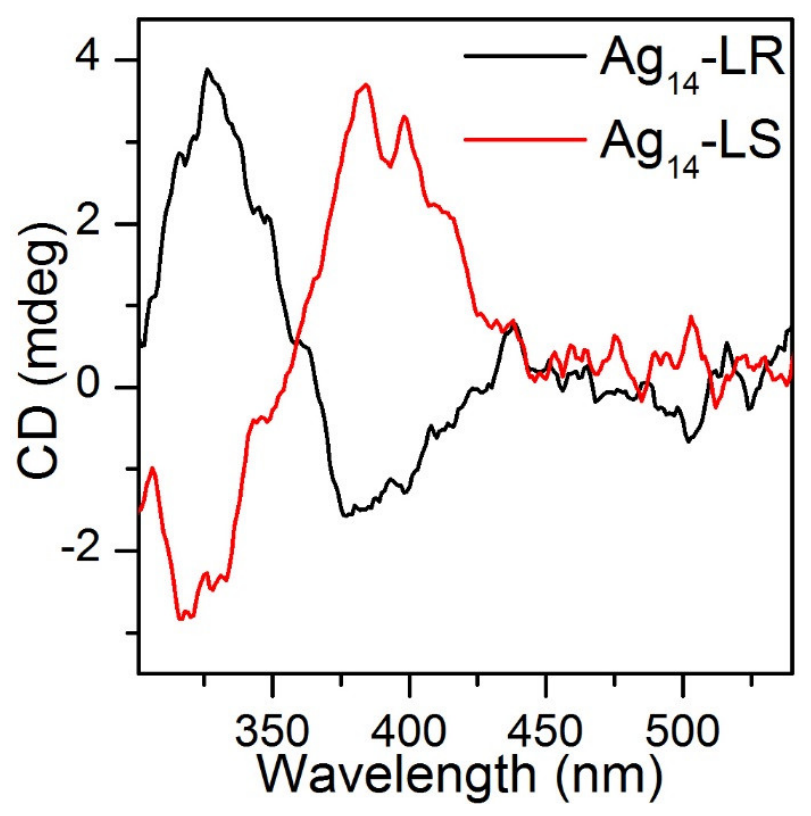

Figure S9. Solid state CD spectra of $\mathbf{A g}_{14}-\mathbf{L S}$ (crystals) and $\mathbf{A g} \mathbf{g}_{14}-\mathbf{L R}$ (crystals).

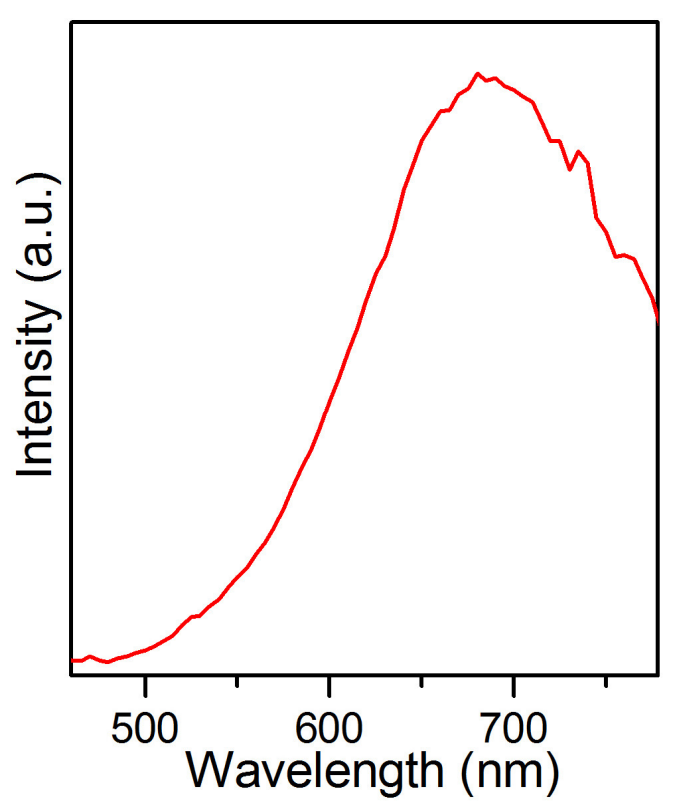

Figure S10. Solid-state fluorescence spectrum of $\mathbf{A g}_{14}-\mathbf{L R}$ at room temperature, excited at $420 \mathrm{~nm}$. 


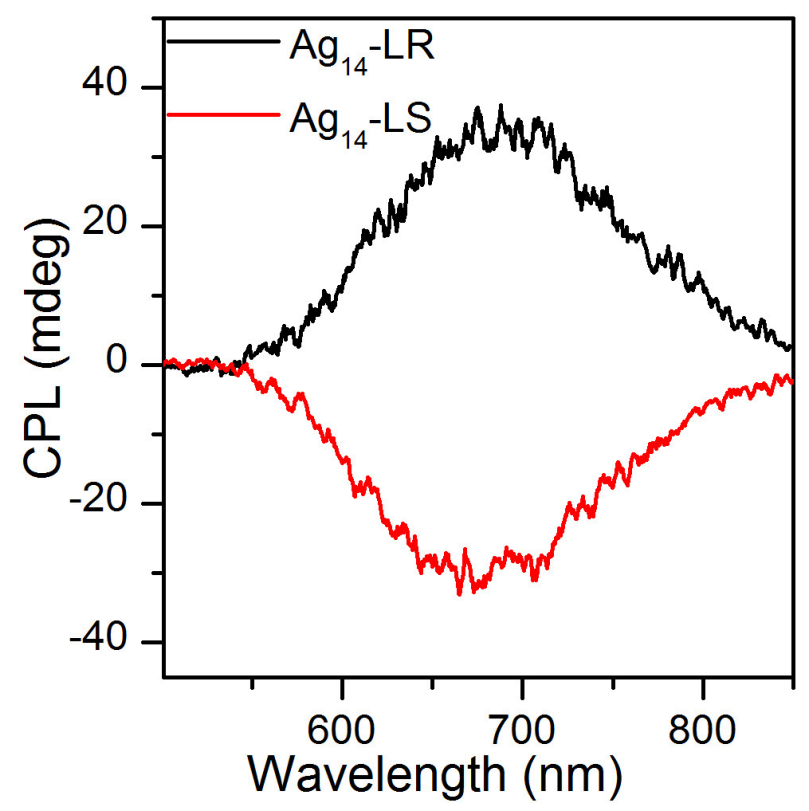

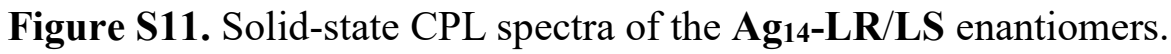

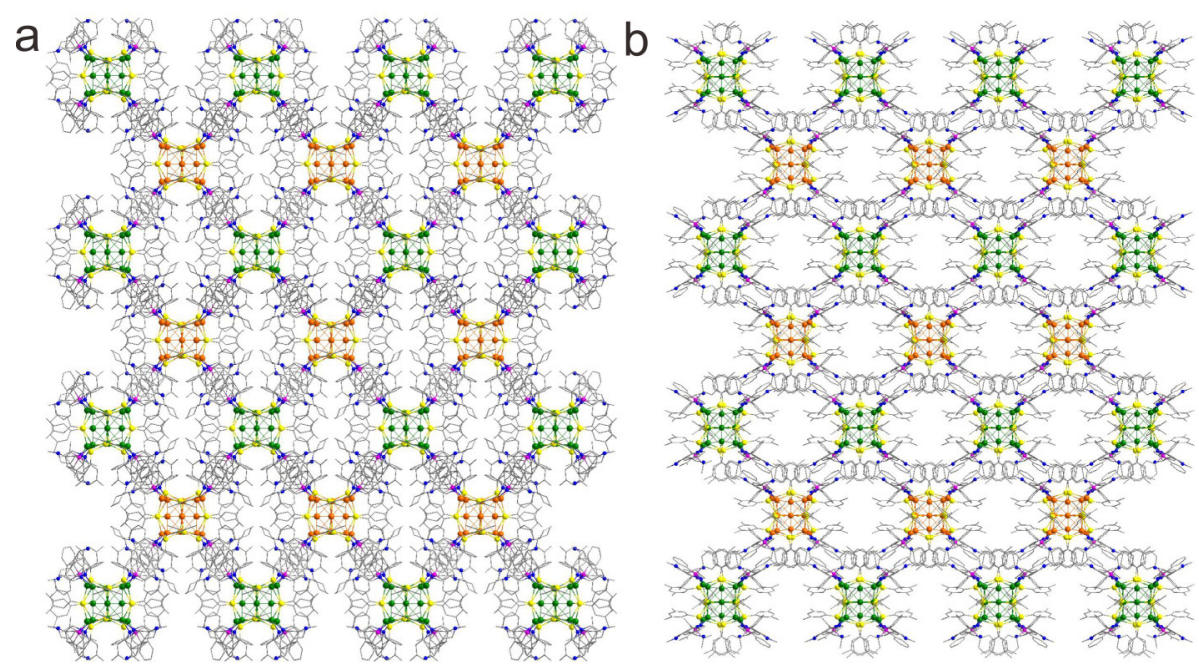

Figure S12. Crystal structure of $\mathbf{A g}_{14}-\mathbf{L R S}$ viewed along $c$ (a) and $b$ axis (b). Color legend: orange and green sphere, Ag; yellow sphere, S; pink sphere, P; blue sphere, N; grey sphere, C. All hydrogen and fluorine atoms are omitted for clarity. 


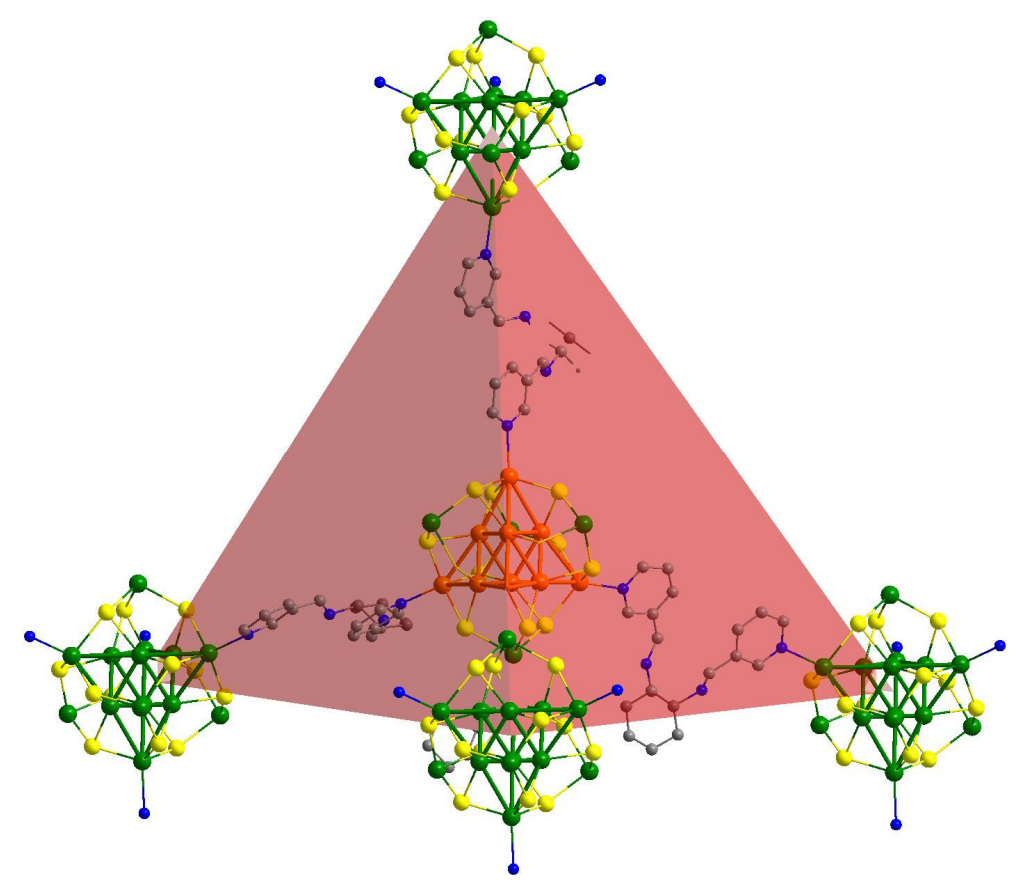

Figure S13. Regular tetrahedron structure formed by the $\mathbf{A g}_{14}$ building blocks (as nodes) in Ag14-LRS. Color legend: orange and green sphere, Ag; yellow sphere, S; blue sphere, N; grey sphere, $\mathrm{C}$.

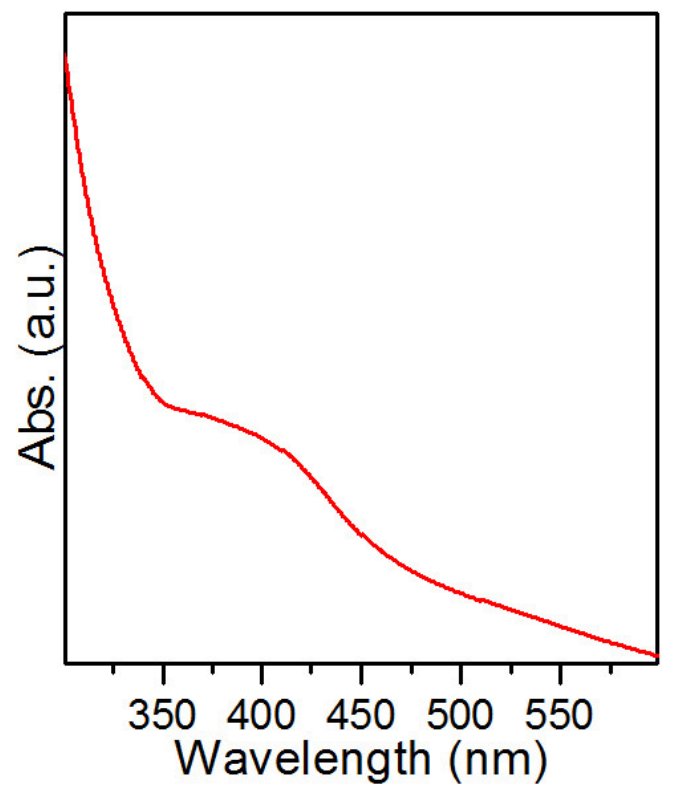

Figure S14. Solid state UV-vis spectrum of $\mathbf{A g}_{14-\mathbf{L R S}}$ (crystals). 


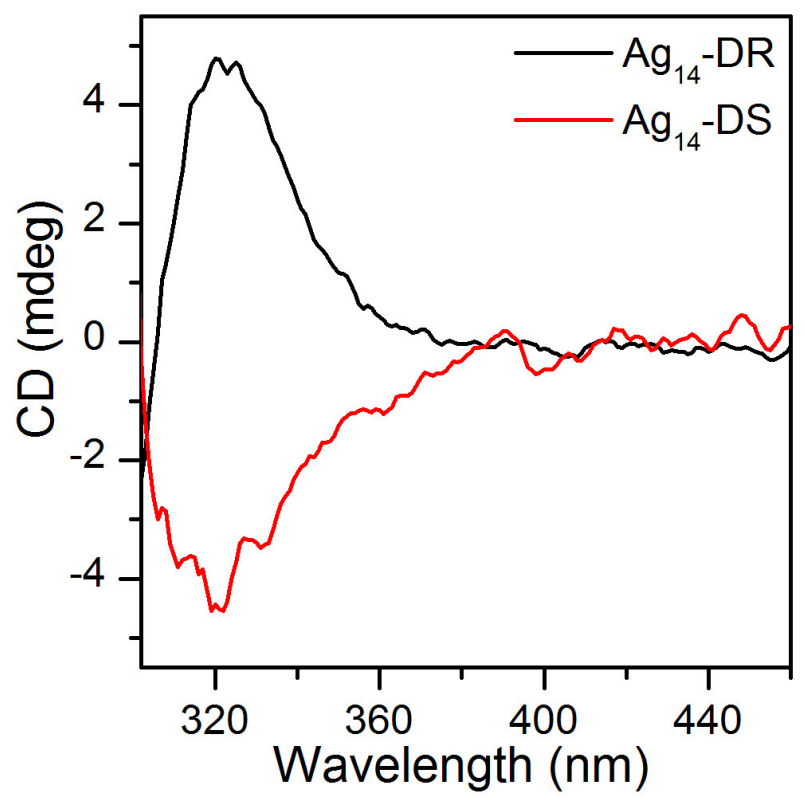

Figure S15. CD spectra of $\mathbf{A g}_{14}-\mathbf{D R}$ and $\mathbf{A g}_{14}-\mathbf{D S}$ in dichloromethane, DMF and methanol mixed solution $(1.0: 0.05: 0.1)$.

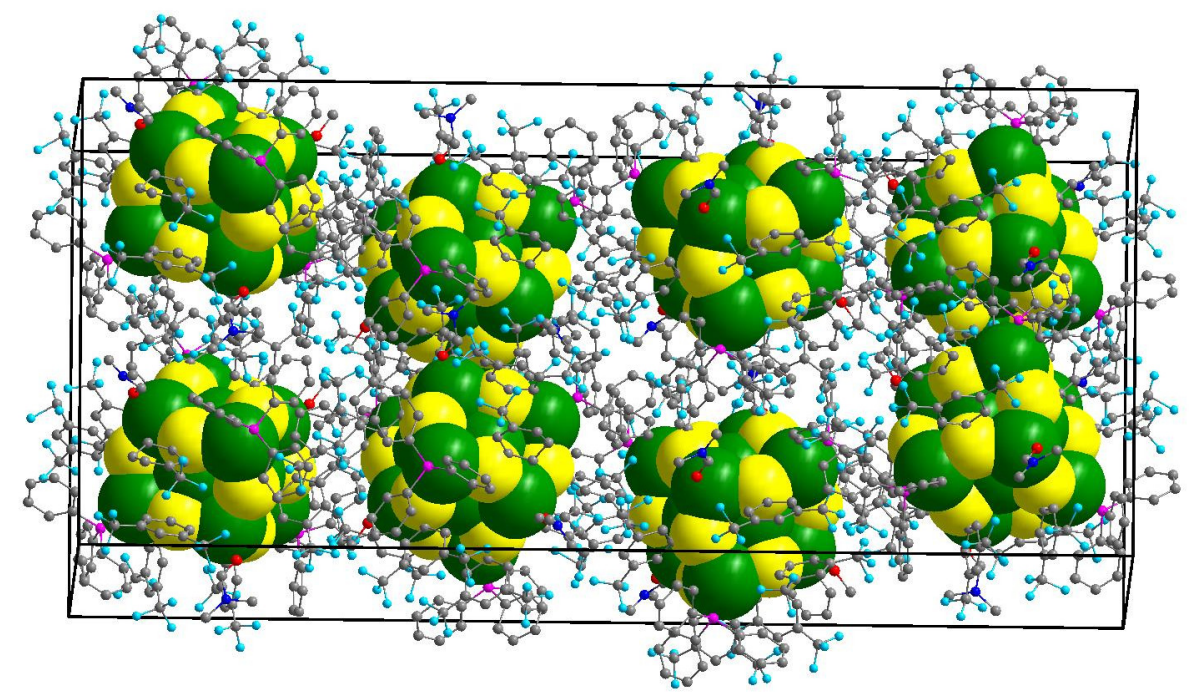

Figure S16. The packing diagram of R-Ag14m. Colour legend: green sphere, Ag; yellow sphere, S; pink sphere, P; red sphere, O; blue sphere, N; sky blue sphere, F; grey sphere, C. All hydrogen atoms are omitted for clarity. 


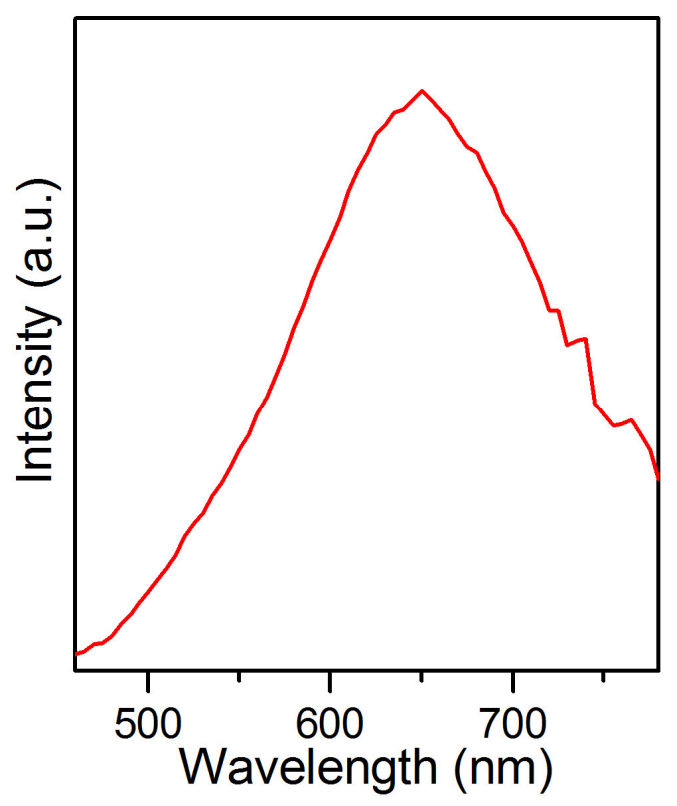

Figure S17. Solid-state fluorescence spectrum of $\mathbf{R}-\mathbf{A g}_{14 \mathrm{~m}}$ at room temperature, excited at $420 \mathrm{~nm}$.

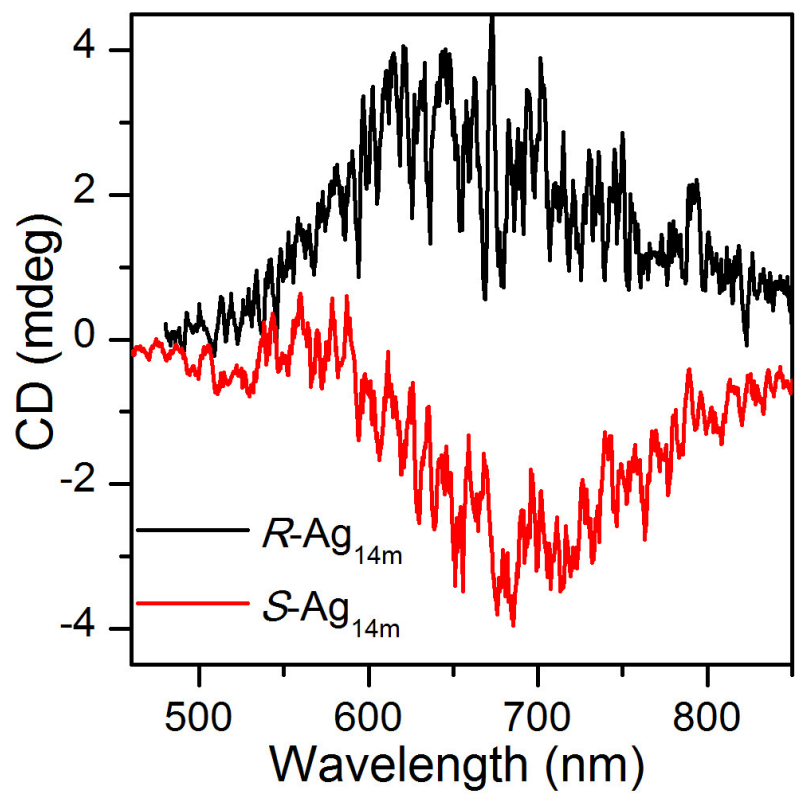

Figure S18. Solid-state CPL spectra of the R/S-Ag14m enantiomers. 


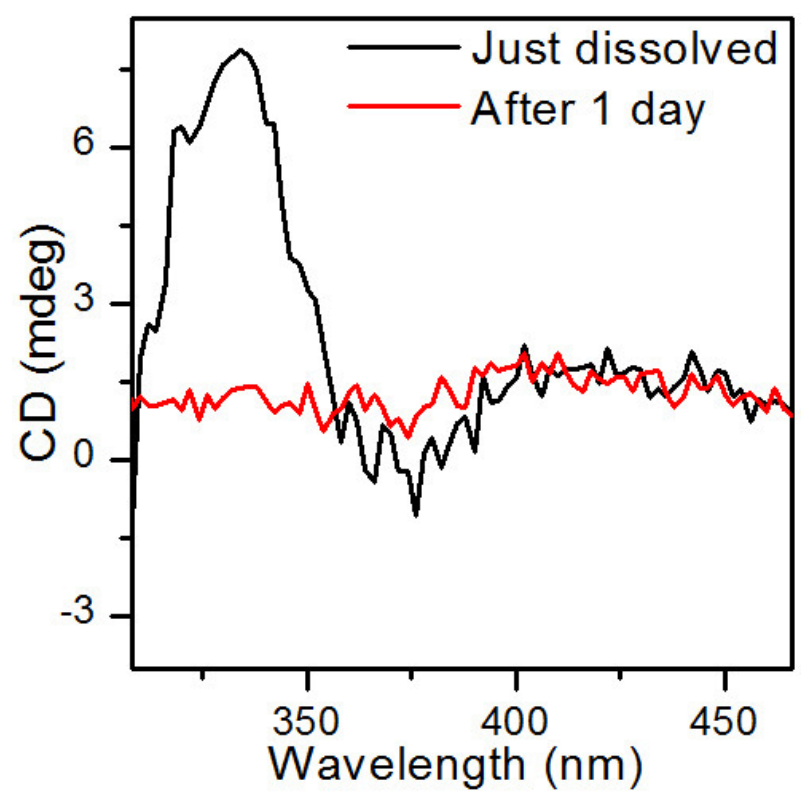

Figure S19. Comparison of the CD spectra of the $\mathbf{R}-\mathbf{A g}_{14 \mathrm{~m}}$ crystals just dissolved in DMF and after 1 day at room temperature.

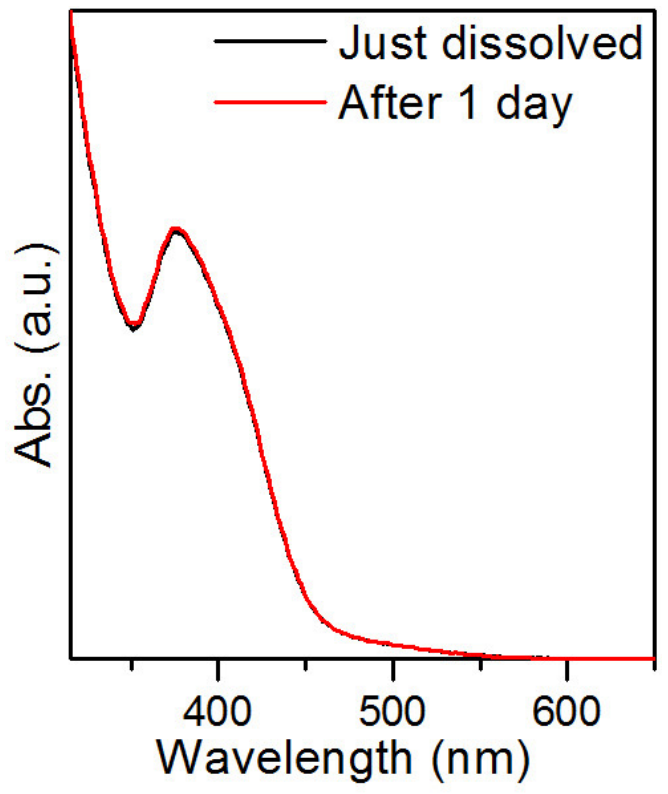

Figure S20. Comparison of the UV-vis spectra of the R-Ag14m crystals just dissolved in DMF and after 1 day at room temperature. 


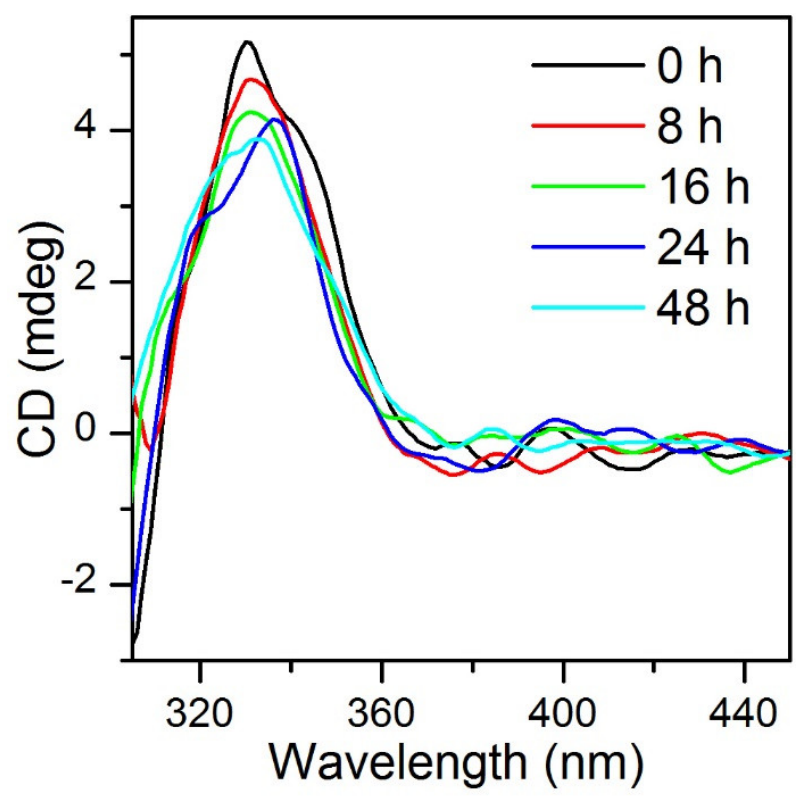

Figure S21. The intensity changes of the CD spectra of $\mathbf{A g}_{14}-\mathbf{D R}$ in dichloromethane, DMF and methanol mixed solution $(1.0: 0.05: 0.1)$ with times at different $25^{\circ} \mathrm{C}$.

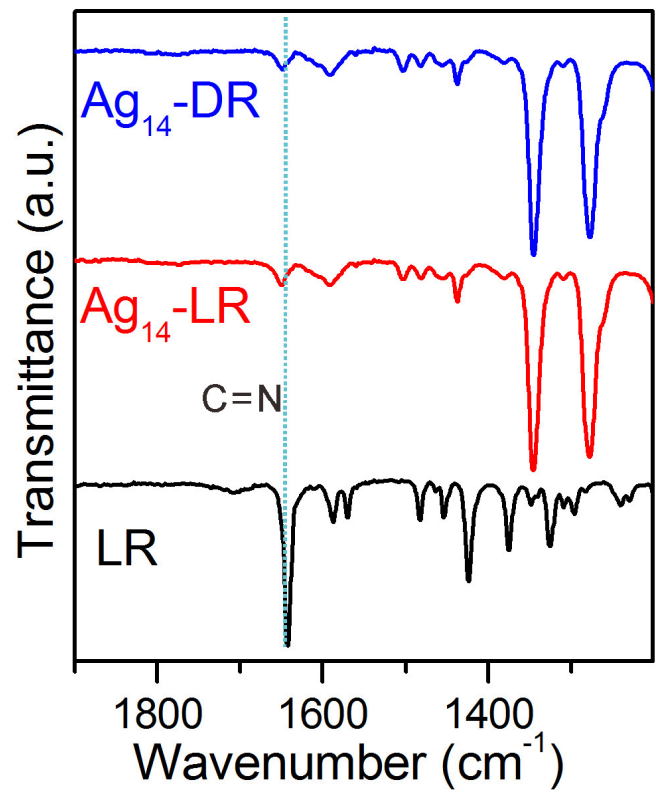

Figure S22. Solid-state FT-IR spectra of $\mathbf{A g}_{14}-\mathbf{D R}$, crystals of $\mathbf{A g}_{14-\mathbf{L R}}$ and LR. 


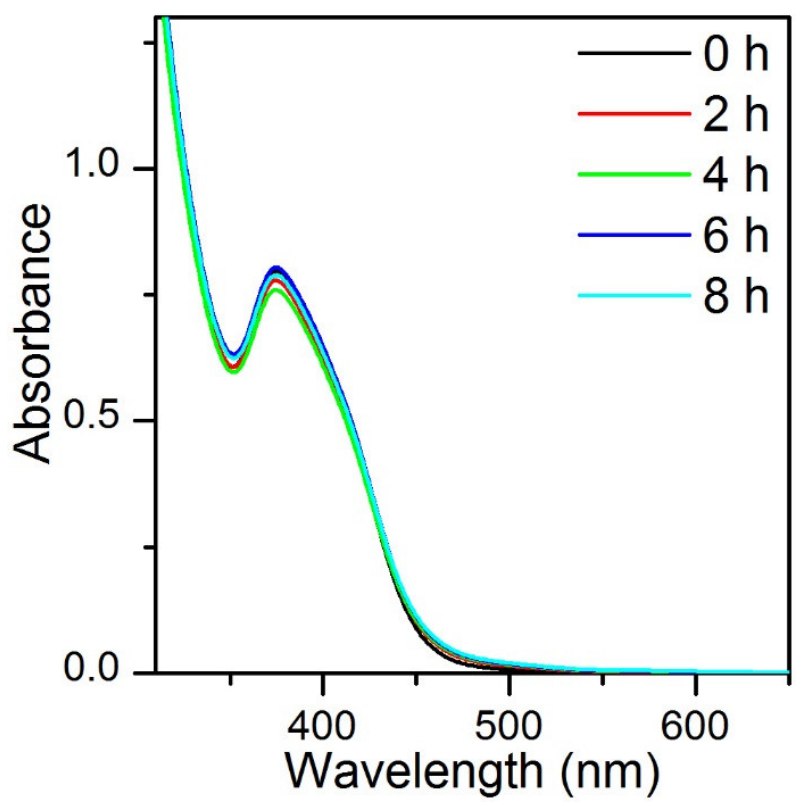

Figure S23. UV-vis spectra of pure R-Ag14m crystals dissolved in DMF during racemization at $30{ }^{\circ} \mathrm{C}$.
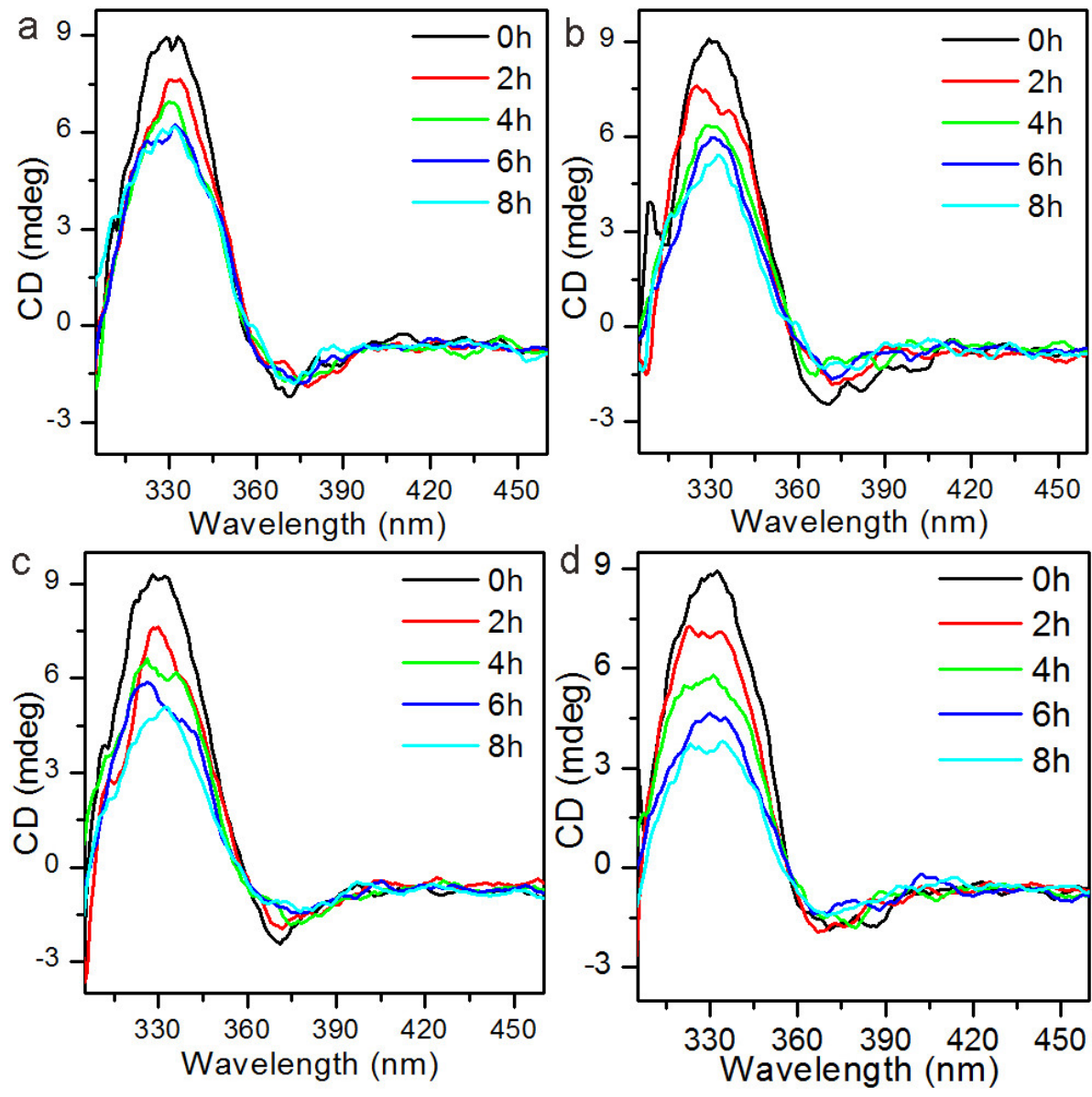

Figure S24. The intensity changes of the CD spectra of R-A $\mathbf{A} \mathbf{g}_{14 \mathrm{~m}}$ crystals in DMF with times at different temperatures: (a) $0{ }^{\circ} \mathrm{C}$; (b) $10{ }^{\circ} \mathrm{C}$; (c) $20{ }^{\circ} \mathrm{C}$; (d) $30{ }^{\circ} \mathrm{C}$. 


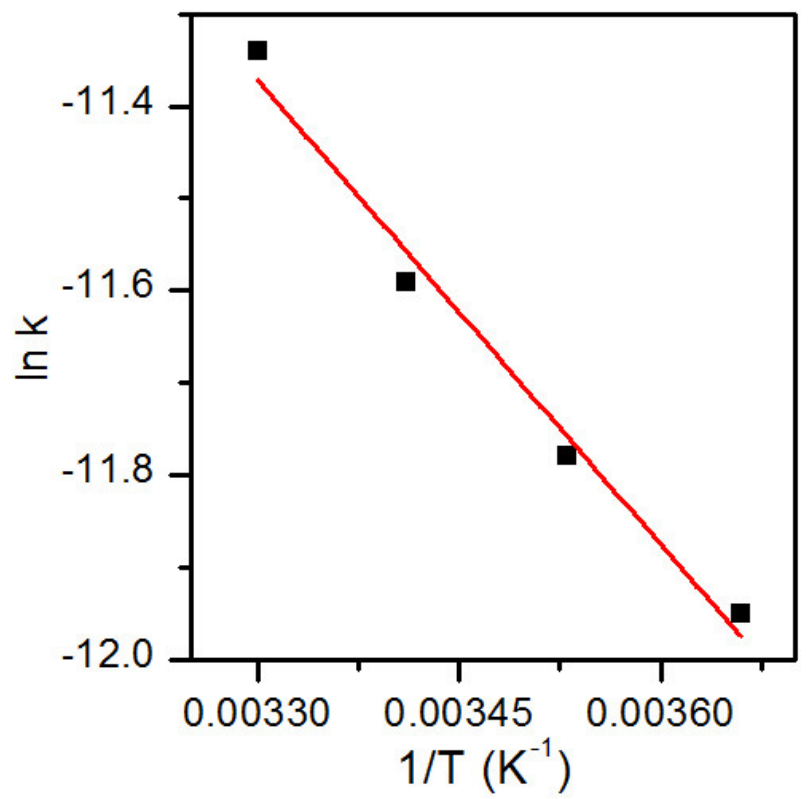

Figure S25. Arrhenius plot $\ln (k)$ vs $1 / \mathrm{T}$ for the auto-racemization of pure crystals of $\mathbf{R}$ Ag14m. 
Table S1. Crystal data and structure refinement for $\mathbf{A g}_{14-\mathbf{L R}}$.

Identification code

Empirical formula

Formula weight

Temperature/K

Crystal system

Space group

$\mathrm{a} / \AA$

$\mathrm{b} / \AA$

$\mathrm{c} / \AA$

$\alpha /{ }^{\circ}$

$\beta /{ }^{\circ}$

$\gamma /{ }^{\circ}$

Volume $/ \AA^{3}$

Z

$\rho_{\text {calc }} / \mathrm{cm}^{3}$

$\mu / \mathrm{mm}^{-1}$

$\mathrm{F}(000)$

Crystal size $/ \mathrm{mm}^{3}$

Radiation

$2 \Theta$ range for data collection $/^{\circ}$

Index ranges

Reflections collected

Independent reflections

Data/restraints/parameters

Goodness-of-fit on $\mathrm{F}^{2}$

Final R indexes $[\mathrm{I}>=2 \sigma(\mathrm{I})]$

Final $\mathrm{R}$ indexes [all data]

Largest diff. peak/hole / e $\AA^{-3}$

Flack parameter

\section{Ag14-LR}

$\mathrm{C}_{204} \mathrm{H}_{136} \mathrm{Ag}_{14} \mathrm{~F}_{72} \mathrm{~N}_{8} \mathrm{P}_{4} \mathrm{~S}_{12}$

6085.98

100.01(10)

hexagonal

P6 122

23.6655(7)

23.6655(7)

$81.7805(17)$

90

90

120

$39665(2)$

6

1.529

10.069

17844.0

$0.02 \times 0.01 \times 0.01$

$\mathrm{Cu} \mathrm{K \alpha}(\lambda=1.54184)$

6.914 to 122.338

$-16 \leq \mathrm{h} \leq 24,-21 \leq \mathrm{k} \leq 18,-88 \leq 1 \leq 92$

77560

$19620\left[\mathrm{R}_{\text {int }}=0.1399, \mathrm{R}_{\text {sigma }}=0.1315\right]$

$19620 / 1564 / 1355$

1.042

$\mathrm{R}_{1}=0.1023, \mathrm{wR}_{2}=0.2235$

$\mathrm{R}_{1}=0.1265, \mathrm{wR}_{2}=0.2442$

$1.51 /-1.67$

$0.018(8)$ 
Table S2. Crystal data and structure refinement for $\mathbf{A g}_{14-\mathbf{L S}}$

Identification code

Empirical formula

Formula weight

Temperature/K

Crystal system

Space group

$\mathrm{a} / \AA$

$\mathrm{b} / \AA$

$\mathrm{c} / \AA$

$\alpha /{ }^{\circ}$

$\beta /{ }^{\circ}$

$\gamma /{ }^{\circ}$

Volume $/ \AA^{3}$

Z

$\rho_{\text {calc }} / \mathrm{cm}^{3}$

$\mu / \mathrm{mm}^{-1}$

$\mathrm{F}(000)$

Crystal size $/ \mathrm{mm}^{3}$

Radiation

$2 \Theta$ range for data collection $/^{\circ}$

Index ranges

Reflections collected

Independent reflections

Data/restraints/parameters

Goodness-of-fit on $\mathrm{F}^{2}$

Final R indexes $[\mathrm{I}>=2 \sigma(\mathrm{I})]$

Final $\mathrm{R}$ indexes [all data]

Largest diff. peak/hole / e $\AA^{-3}$

Flack parameter

\section{Ag14-LS}

$\mathrm{C}_{204} \mathrm{H}_{136} \mathrm{Ag}_{14} \mathrm{~F}_{72} \mathrm{~N}_{8} \mathrm{P}_{4} \mathrm{~S}_{12}$

6085.98

100.01(10)

hexagonal

$\mathrm{P}_{5} 22$

23.6036(4)

23.6036(4)

$80.9175(11)$

90

90

120

39041.8(14)

6

1.553

10.229

17844.0

$0.03 \times 0.02 \times 0.02$

$\mathrm{Cu} \mathrm{K \alpha}(\lambda=1.54184)$

6.966 to 122.338

$-26 \leq \mathrm{h} \leq 26,-26 \leq \mathrm{k} \leq 26,-91 \leq 1 \leq 91$

156944

$20006\left[\mathrm{R}_{\text {int }}=0.1401, \mathrm{R}_{\text {sigma }}=0.0703\right]$

20006/1002/1415

1.069

$\mathrm{R}_{1}=0.0892, \mathrm{wR}_{2}=0.1872$

$\mathrm{R}_{1}=0.1013, \mathrm{wR}_{2}=0.1959$

$1.40 /-1.13$

$0.055(5)$ 
Table S3. Crystal data and structure refinement for Ag14-LRS.

Identification code

Empirical formula

Formula weight

Temperature/K

Crystal system

Space group

$\mathrm{a} / \AA$

$\mathrm{b} / \AA$

$\mathrm{c} / \AA$

$\alpha /{ }^{\circ}$

$\beta /{ }^{\circ}$

$\gamma /{ }^{\circ}$

Volume $/ \AA^{3}$

Z

$\rho_{\text {calc }} / \mathrm{cm}^{3}$

$\mu / \mathrm{mm}^{-1}$

$\mathrm{F}(000)$

Crystal size $/ \mathrm{mm}^{3}$

Radiation

$2 \Theta$ range for data collection/ ${ }^{\circ}$

Index ranges

Reflections collected

Independent reflections

Data/restraints/parameters

Goodness-of-fit on $\mathrm{F}^{2}$

Final R indexes $[\mathrm{I}>=2 \sigma(\mathrm{I})]$

Final $\mathrm{R}$ indexes [all data]

Largest diff. peak/hole / e $\AA^{-3}$

\section{Ag14-LRS}

$\mathrm{C}_{204} \mathrm{H}_{136} \mathrm{Ag}_{14} \mathrm{~F}_{72} \mathrm{~N}_{8} \mathrm{P}_{4} \mathrm{~S}_{12}$

6085.98

100.00(10)

orthorhombic

Pnnn

20.4683(3)

24.1337(4)

$25.9145(6)$

90

90

90

12801.1(4)

2

1.579

10.400

5948.0

$0.02 \times 0.01 \times 0.01$

$\mathrm{Cu} \mathrm{K \alpha}(\lambda=1.54184)$

7.326 to 122.338

$-23 \leq \mathrm{h} \leq 20,-27 \leq \mathrm{k} \leq 20,-27 \leq 1 \leq 29$

38362

$9835\left[\mathrm{R}_{\text {int }}=0.0693, \mathrm{R}_{\text {sigma }}=0.0529\right]$

9835/752/784

1.017

$\mathrm{R}_{1}=0.0616, \mathrm{wR}_{2}=0.1627$

$\mathrm{R}_{1}=0.0909, \mathrm{wR}_{2}=0.1930$

$1.63 /-1.00$ 
Table S4. Crystal data and structure refinement for $\mathbf{R}-\mathbf{A g} \mathbf{1 4 m}$.

Identification code

Empirical formula

Formula weight

Temperature/K

Crystal system

Space group

$\mathrm{a} / \AA$

$\mathrm{b} / \AA$

$\mathrm{c} / \AA$

$\alpha /{ }^{\circ}$

$\beta /{ }^{\circ}$

$\gamma /{ }^{\circ}$

Volume $/ \AA^{3}$

Z

$\rho_{\text {calc }} / \mathrm{cm}^{3}$

$\mu / \mathrm{mm}^{-1}$

$\mathrm{F}(000)$

Crystal size $/ \mathrm{mm}^{3}$

Radiation

$2 \Theta$ range for data collection $/^{\circ}$

Index ranges

Reflections collected

Independent reflections

Data/restraints/parameters

Goodness-of-fit on $\mathrm{F}^{2}$

Final R indexes $[\mathrm{I}>=2 \sigma(\mathrm{I})]$

Final $\mathrm{R}$ indexes [all data]

Largest diff. peak/hole / e $\AA^{-3}$

Flack parameter

\section{R-Ag14m}

$\mathrm{C}_{178} \mathrm{H}_{121} \mathrm{Ag}_{14} \mathrm{~F}_{72} \mathrm{~N}_{3} \mathrm{O}_{4} \mathrm{P}_{4} \mathrm{~S}_{12}$

5752.55

100.01(10)

orthorhombic

C2221

24.9972(3)

30.3589(4)

56.1233(7)

90

90

90

42591.3(9)

8

1.794

12.465

22400.0

$0.05 \times 0.02 \times 0.02$

$\mathrm{Cu} \mathrm{K \alpha}(\lambda=1.54184)$

6.58 to 122.338

$-25 \leq \mathrm{h} \leq 28,-34 \leq \mathrm{k} \leq 34,-63 \leq 1 \leq 63$

129060

$32755\left[\mathrm{R}_{\text {int }}=0.1137, \mathrm{R}_{\text {sigma }}=0.0904\right]$

$32755 / 1869 / 2570$

1.058

$\mathrm{R}_{1}=0.0790, \mathrm{wR}_{2}=0.1977$

$\mathrm{R}_{1}=0.0944, \mathrm{wR}_{2}=0.2110$

$1.92 /-1.29$

$-0.026(5)$ 
Table S5. Crystal data and structure refinement for $\mathbf{S}-\mathbf{A g} \mathbf{1 4 m}$.

Identification code

Empirical formula

Formula weight

Temperature/K

Crystal system

Space group

$\mathrm{a} / \AA$

$\mathrm{b} / \AA$

$\mathrm{c} / \AA$

$\alpha /{ }^{\circ}$

$\beta /{ }^{\circ}$

$\gamma /{ }^{\circ}$

Volume $/ \AA^{3}$

Z

$\rho_{\text {calc }} / \mathrm{cm}^{3}$

$\mu / \mathrm{mm}^{-1}$

$\mathrm{F}(000)$

Crystal size $/ \mathrm{mm}^{3}$

Radiation

$2 \Theta$ range for data collection $/^{\circ}$

Index ranges

Reflections collected

Independent reflections

Data/restraints/parameters

Goodness-of-fit on $\mathrm{F}^{2}$

Final R indexes $[\mathrm{I}>=2 \sigma(\mathrm{I})]$

Final $\mathrm{R}$ indexes [all data]

Largest diff. peak/hole / e $\AA^{-3}$

Flack parameter

\section{S-Ag14m}

$\mathrm{C}_{178} \mathrm{H}_{121} \mathrm{Ag}_{14} \mathrm{~F}_{72} \mathrm{~N}_{3} \mathrm{O}_{4} \mathrm{P}_{4} \mathrm{~S}_{12}$

5752.55

100.01(10)

orthorhombic

C2221

24.8562(2)

30.5304(3)

$56.0821(5)$

90

90

90

42559.1(7)

8

1.796

12.475

22400.0

$0.04 \times 0.02 \times 0.02$

$\mathrm{Cu} \mathrm{K \alpha}(\lambda=1.54184)$

6.586 to 122.334

$-28 \leq \mathrm{h} \leq 16,-34 \leq \mathrm{k} \leq 33,-63 \leq 1 \leq 48$

69042

$32631\left[\mathrm{R}_{\text {int }}=0.0594, \mathrm{R}_{\text {sigma }}=0.0606\right]$

$32631 / 1935 / 2557$

1.057

$\mathrm{R}_{1}=0.0692, \mathrm{wR}_{2}=0.1759$

$\mathrm{R}_{1}=0.0712, \mathrm{wR}_{2}=0.1776$

$2.21 /-2.33$

$0.015(5)$ 